PROCEEDINGS OF THE

AMERICAN MATHEMATICAL SOCIETY

Volume 32, Numter 2, April 1972

\title{
A NOTE ON HOMOTOPY EQUIVALENCES
}

\author{
R. M. VOGT
}

Abstract. Given a homotopy equivalence $f: X \rightarrow Y$, a homotopy inverse $g$ of $f$, and a homotopy $H: X \times I \rightarrow X$ from $g \circ f$ to $1_{X}$. We show that there is a homotopy $K: Y \times I \rightarrow Y$ from $f \circ g$ to $1_{Y}$ such that $f \circ H \simeq K \circ\left(f \times 1_{I}\right)$ rel $X \times \partial I$ and $H \circ\left(g \times 1_{I}\right) \simeq g \circ K$ rel $Y \times \partial I$.

In [1], R. Lashof introduced the notion of a strong homotopy equivalence in context with the study of reductions of topological microbundles to piecewise linear or differentiable microbundles.

Definition. A strong homotopy equivalence between two spaces $X$ and $Y$ is a quadruple $(f, g, H, K)$ where $f: X \rightarrow Y$ and $g: Y \rightarrow X$ are maps and $H: X \times I \rightarrow X$ and $K: Y \times I \rightarrow Y$ are homotopies, $H: g \circ f \simeq 1_{X}, K$ : $f \circ g \simeq 1_{Y}$, such that $f \circ H \simeq K \circ\left(f \times 1_{I}\right)$ rel endpoints and $H \circ\left(g \times 1_{I}\right) \simeq g \circ K$ rel endpoints (here $I$ denotes the unit interval).

Now the question arises, whether any homotopy equivalence can be made into a strong one. The following proposition gives an affirmative answer. The result is also quite useful for many questions in homotopy theory. Although it is implicitly contained in various papers, I have never found it stated explicitly. The proof is very elementary, only using the basic facts about track addition of homotopies.

Proposition. Let $f: X \rightarrow Y$ be a homotopy equivalence with homotopy inverse $g$. Let $H: X \times I \rightarrow X$ be a homotopy from $g \circ f$ to $1_{X}$. Then there exists a homotopy $K: Y \times I \rightarrow Y$ from $f \circ g$ to $1_{Y}$ such that $f \circ H \simeq K \circ\left(f \times 1_{I}\right)$ rel endpoints and $H \circ\left(g \times 1_{I}\right) \simeq g \circ K$ rel endpoints.

Let $[X, Z]_{p}^{q}$ denote the set of equivalence classes of homotopies $F: X \times I \rightarrow Z$ such that $F \mid X \times 0=p: X \rightarrow Z$ and $F \mid X \times 1=q: X \rightarrow Z$. Two such homotopies are defined to be equivalent if they are homotopic rel endpoints.

Maps $h: X \rightarrow Y$ and $k: Z \rightarrow W$ induce maps $h^{*}:[Y, Z]_{p}^{q} \rightarrow[X, Z]_{p \circ h}^{q_{o} h}$, given by $h^{*}[M]=[M \circ(h \times 1)]$, where $[M]$ denotes the equivalence class of $M$, and $k_{*}:[X, Z]_{u}^{v} \rightarrow[X, W]_{k \circ u}^{k \circ v}$, given by $k_{*}[N]=[k \circ N]$.

Lemma 1. Let $[R] \in[U, V]_{k}^{l}$ and $[Q] \in[V, W]_{u}^{v}$. Then

$$
[u \circ R+Q \circ(l \times 1)]=[Q \circ(k \times 1)+v \circ R]
$$

Received by the editors August 19, 1970.

AMS 1970 subject classifications. Primary 55D10.

(c) American Mathematical Society 1972 
in $[U, W]_{u \circ k}^{v_{0} l}$, where " + " denotes track addition, the left homotopy is applied first.

Proof. The map $G: U \times I \times I \rightarrow W$, given by $G\left(x, t_{1}, t_{2}\right)=Q\left(R\left(x, t_{1}\right), t_{2}\right)$ is the required homotopy rel endpoints between $u \circ R+Q \circ(l \times 1)$ and $Q \circ(k \times 1)+v \circ R$, because $G|U \times 0 \times I=Q \circ(k \times 1), g| U \times 1 \times I=Q \circ(l \times 1)$, $g|U \times I \times 0=u \circ R, G| U \times I \times 1=v \circ R$.

Lemma 2. With the notation of the proposition, $f^{*}:[Y, Z]_{p}^{q} \rightarrow[X, Z]_{p_{\circ} f}^{q \circ f}$ and $g^{*}:[X, Z]_{u}^{v} \rightarrow[Y, Z]_{u_{\circ} g}^{v_{\circ} g}$ are bijections.

Proof. Consider the composite

$$
r:[Y, Z]_{p}^{q} \stackrel{f^{*}}{\longrightarrow}[X, Z]_{p_{\circ} f}^{q_{\circ} f} \stackrel{g^{*}}{\longrightarrow}[Y, Z]_{p_{\circ} f_{\circ} g}^{q_{\circ} \circ g} \stackrel{s}{\longrightarrow}[Y, Z]_{q}^{a}
$$

where $s$ is given by $s[M]=[-p \circ F+M+q \circ F]$, and $F: f \circ g \simeq 1_{Y}$ is an arbitrary fixed homotopy. For $[Q] \in[Y, Z]_{p}^{q}$, we have

$$
r[Q]=[-p \circ F+Q \circ(f \circ g \times 1)+q \circ F] .
$$

Substituting $R$ by $F$ in the previous lemma, we find that $r[Q]=[Q]$. Since $s$ is a bijection (with inverse $[N] \mapsto\left[p \circ F+N-q_{\circ} F\right]$ ), the composite $g^{*} \circ f^{*}$ is a bijection, and hence $f^{*}$ injective and $g^{*}$ surjective. Analogously one shows that in the sequence

$$
[X, Z]_{u}^{v} \stackrel{g^{*}}{\longrightarrow}[Y, Z]_{u \circ g}^{v \circ g} \stackrel{f^{*}}{\longrightarrow}[X, Z]_{u_{0 \circ} \circ f}^{v_{\circ} g_{\circ} f}
$$

$g^{*}$ is injective and $f^{*}$ is surjective. Hence, in $\left(^{*}\right), g^{*}$ and therefore $f^{*}$ are bijective. Similarly in $\left(^{* *}\right), f^{*}$ and hence $g^{*}$ are bijective.

Proof of the Proposition. We are given $H: g \circ f \simeq 1_{X}$. Choose $K$ to be a representative of $f^{*-1}[f \circ H] \in[Y, Y]_{f_{\circ} g}^{1}$, and let $H^{\prime}$ be a representative of $g^{*-1}[g \circ K] \in[X, X]_{g \circ f}^{1}$. Then $K \circ(f \times 1) \simeq f \circ H$ rel endpoints, and $H^{\prime}$ $\circ(g \times 1) \simeq g \circ K$ rel endpoints. Consider the composite

$$
r:[Y, X]_{g_{\circ} f_{\circ} g}^{g} \stackrel{f_{*}}{\longrightarrow}[Y, Y]_{f_{\circ} g_{\circ} \circ g}^{f_{\circ} g} \stackrel{s}{\longrightarrow}[Y, Y]_{f_{\circ} g}^{1} \stackrel{g_{*}}{\longrightarrow}[Y, X]_{g_{\circ} f_{\circ} g}^{g}
$$

where $s$ is defined by $s[M]=[-K \circ(f \circ g \times 1)+M+K]$. Let $[R] \in[Y, X]_{g_{\circ} \circ \circ \text {, }}^{g}$, then

$$
\begin{aligned}
r[R] & =[-g \circ K \circ(f \circ g \times 1)+g \circ f \circ R+g \circ K] \\
& =\left[-H^{\prime} \circ(g \circ f \circ g \times 1)+g \circ f \circ R+H^{\prime} \circ(g \times 1)\right]=[R] .
\end{aligned}
$$

The last equality follows from Lemma 1 by substituting $Q$ by $H^{\prime}$. 
Now $[H \circ(g \times 1)] \in[Y, X]_{g_{\circ} \circ g}^{g}$, and therefore

$$
\begin{aligned}
{[H \circ(g \times 1)] } & =r[H \circ(g \times 1)] \\
& =[-g \circ K \circ(f \circ g \times 1)+g \circ f \circ H \circ(g \times 1)+g \circ K] \\
& =[-g \circ K \circ(f \circ g \times 1)+g \circ K \circ(f \circ g \times 1)+g \circ K] \\
& =[g \circ K] .
\end{aligned}
$$

So $H \circ(g \times 1) \simeq g \circ K$ rel endpoints.

\section{REFERENCES}

1. R. Lashof, The immersion approach to triangulation and smoothing, Proc. Advanced Study Institute on Algebraic Topology, Mathematisk Institut, Aarhus Universitet, Aarhus, 1970.

\section{Matematisk Institut, Aarhus Universitet, Aarhus, Denmark}

Current address: Mathematisches Institut, Universität des Saarlandes, Saarbrücken, Federal Republic of Germany 